\title{
Apparent life threatening events and infant holding practices
}

\author{
Roger W Byard, Richard H Burnell
}

\begin{abstract}
Two 4 week old infants are reported who suffered apparent life threatening events (ALTE) while being cradled in adult arms. The events, which could be reproduced under controlled circumstances, were not associated with any struggling by the infants. Alteration of infant holding practices in both cases resulted in cessation of the events. A case of cardiorespiratory arrest during breast feeding in an 8 week old infant that was unnoticed by her mother is also described. These cases suggest that certain infants may not respond normally to airway occlusion while being held or nursed, and show that careful study of the events surrounding ALTE may reveal contributing environmental factors. If ALTE occur around feeding time, observation of how caretakers place the infant during and after feeding may be informative.

(Arch Dis Child 1995; 73: 502-504)
\end{abstract}

Keywords: apnoea, airway obstruction, apparent life threatening event, SIDS.

Airway obstruction is a recognised cause of apparent life threatening events (ALTE) and sudden unexpected death in infancy. The following cases provide evidence that some infants may fail to respond normally to accidental occlusion of the nose and mouth when being either incorrectly held in the arms of an adult, or being breast fed. Careful investigation of the circumstances of ALTE may be crucial in determining possible contributing environmental factors and underlying mechanisms.

\section{Case reports}

CASE 1

A 4 week old boy, who had been born at 37 weeks' gestation, was investigated at a country hospital following two episodes of apnoea with cyanosis. Each episode had occurred in the early hours of the morning approximately 20 minutes after breast feeding and had required mouth to mouth resuscitation by the maternal grandmother. The infant had been otherwise well and thriving, although significant gastrooesophageal reflux was documented by barium swallow and a small ventricular septal defect was confirmed on echocardiography. $\mathrm{He}$ remained well for the week-long hospital admission. The day after discharge a similar event occurred at 2.00 am while being held in his grandmother's arms, resulting in referral to the Adelaide Children's Hospital for monitoring and further investigation. He remained well for the 5 day admission, with a normal overnight pneumogram. The day after discharge, however, a similar event requiring resuscitation again occurred at 2.00 am with his grandmother.

In reconstructing events around early morning feeding it transpired that the grandmother would also rise with her daughter and would hold the infant in her arms after feeding had finished. On the first two occasions the mother had returned from making tea to find the infant's feet blue. The remainder of the infant was not visible as he was being clasped under his grandmother's ample breast in a fold of a large quilted polyester dressing gown.

Reconstruction of the events in the office of one us (RHB) clearly showed obstruction of the infant's nose and mouth, with resultant apnoea and mild cyanosis. This occurred when the infant was held in the usual position by his grandmother wearing her quilted dressing gown. The infant had been fed and was asleep at the time of the supervised ALTE and made no attempts to breathe or struggle at any time. Following identification of the cause of the ALTE, the grandmother altered her pattern of infant holding and no further episodes have occurred. The child is now 6 years old with no medical problems.

\section{CASE 2}

A 4 week old boy, who had been born at 38 weeks' gestation, was admitted to the Adelaide Children's Hospital following a prolonged period of apnoea that had been labelled as a 'near-miss SIDS' episode. Extensive investigations including chest $x$ ray, electrocardiogram, and an overnight pneumogram were undertaken. These failed to reveal any abnormalities. No apnoeic episodes occurred in hospital.

Following interview with one us (RHB) it was discovered that, just as in the previous case, the ALTE had occurred while the infant was being held firmly in his grandmother's arms. His grandmother also had large breasts and wore a quilted dressing gown.

A similar supervised event reconstruction was undertaken with the grandmother and infant which showed that the deeply sleeping infant made no effort to breathe or struggle while being sufficiently obstructed to become mildly cyanotic. The experiment was terminated at this point. Again, following identification of the cause of the ALTE, the grandmother altered her pattern of infant holding and no further episodes have occurred. The child is now 5 years old, with no medical problems. 
CASE 3

An 8 week old girl was noted by her mother to be limp and unresponsive while being breast fed. The infant had been held firmly with her face to the mother's left breast with the nipple in her mouth while her mother sat on the floor and leaned forward to watch another child through a window. Resuscitation was attempted with only partial response and brain death was diagnosed in hospital 2 days later. The only significant clinical history was of three episodes in an obstetric hospital, soon after delivery, of cyanotic episodes associated with apnoea while breast feeding. One episode was witnessed by nursing staff. Respiration restarted after each episode once the infant was removed from the breast. There had been no further episodes since discharge from hospital.

At necropsy the infant was small (length and weight below the third centile) but normally formed. There was no evidence of congenital cardiovascular, pulmonary, or central nervous system disease except for a small $(2 \mathrm{~mm}$ diameter) patent ductus arteriosus not associated with cardiomegaly. All organs were normal except for mild microvesicular steatosis of the liver and diffuse hypoxic-ischaemic encephalopathy of the brain. Although death was attributed to hypoxic-ischaemic encephalopathy secondary to cardiorespiratory arrest of unknown cause, circumstantial evidence implicated failure to respond to possible airway occlusion.

\section{Discussion}

The possibility that inappropriate holding of infants results in airway obstruction gains support from the reported ALTE occurring in two apparently healthy infants while being cradled in their grandmothers' arms. Both infants had cyanotic episodes successfully reproduced under careful medical supervision while being held by their grandmothers, who were wearing quilted dressing gowns. In each case the infant's face was, at the time of the ALTE, being pushed firmly - albeit inadvertently - against large breasts covered by thick clothing. Neither infant struggled or made any attempt to breathe while being unintentionally suffocated. The concerned reactions of the grandmothers when confronted with the cause of the ALTE, and the absence of similar episodes in other family members left in the care of these women either in the past or subsequently, argued against a diagnosis of Munchausen syndrome by proxy. ${ }^{1}$

Although reconstruction of the events surrounding the ALTE in these cases provided evidence implicating mechanical airway occlusion rather than an occult medical condition, the infants' lack of response to airway occlusion was unusual. Whether this reflected an underlying abnormality in the two infants which interfered with the normal response to airway blockage is uncertain. Reduction in arousal response to partial airway occlusion has been demonstrated in sleeping infants ${ }^{2}$ and thus may also have played a role in the ALTE occurring in these infants. Carbon dioxide accumulation with rebreathing ${ }^{3}$ is another theoretical mechanism which may have contributed to the ALTE in cases 1 and 2, given that both had their faces pushed into bulky clothing. Neither infant had any further episodes once the problems with holding practices had been rectified.

The cardiorespiratory arrest of a 2 month old infant while breast feeding in her mother's arms is also unusual. Subsequent investigation revealed that she had been held to her mother's chest while her mother had been sitting on the floor, stretching forward to look out of a window at an older child playing outside. Although death was attributed to hypoxic-ischaemic encephalopathy secondary to cardiorespiratory arrest of unknown cause there is circumstantial evidence implicating failure to respond to possible airway obstruction from breast feeding. The previous history of cyanotic episodes while being breast fed on at least three occasions is also supportive of ALTE triggered by airway obstruction occurring in this position. Unfortunately for the pathologist, the necropsy findings in cases of accidental or non-accidental airway obstruction in infancy are often identical to those found in sudden infant death syndrome. ${ }^{45}$ For this reason a proper postmortem assessment of such infant deaths requires review of the clinical history and death scene investigation. ${ }^{6}$ Thus, although a 'SIDS-like' event cannot be excluded in case 3 , the circumstances surrounding the terminal episode are not typical.

The issue of parents sleeping in the same bed as their infants has recently been widely debated. Although it has been proposed that bed sharing may reduce the risk of SIDS, ${ }^{7}$ the New Zealand cot death study has shown an increased risk of SIDS in infants of bed sharing mothers who smoke. ${ }^{8}$ While this study found no data to support overlaying or holding practices as causes of death, an early study by Abramson attributed death due to overlaying in 19 out of 139 infants. ${ }^{9}$ It was claimed that death had resulted from occlusion of the nose and mouth of these infants while they were pressed against the breast or arm of mothers who had fallen asleep. Unfortunately lack of detailed death scene descriptions prevents reassessment of these cases. The possibility of maternal sedation as a contributing factor to accidental infantile asphyxiation was shown by the case of a 23 year old mother who fell asleep in hospital while breast feeding. Her 2 day old boy was later found by nursing staff to be unresponsive beneath her right breast. A coronor's inquest attributed death to asphyxia due to overlaying, a contributing factor being maternal drowsiness because of sedative ingestion. ${ }^{10}$

An investigation of the circumstances surrounding death is necessary in all cases of unexpected infant death. A similar investigation or reconstruction of events may also provide additional information which might not otherwise be available in cases of ALTE. This could take the form of a carefully supervised re-enactment of the usual situation in which 
ALTE have occurred, with continued close observation by an attending paediatrician with resuscitation equipment at hand. The possibility of inappropriate positioning, or of failure of normal response to airway blockage, should be considered in these cases. If ALTE occur around feeding time, observation of how caretakers place the infant during and after feeding may be informative.

We thank Ms Maxine Need, SIDS Association of South Australia, for information in case 3.

1 Byard RW, Beal SM. Munchausen syndrome by proxy, infantile apnoea and homicide. I Paediatr Child Health 1993; 29: 77-9.

2 Newman NM, Frost JK, Bury L, Jordan K, Phillips K. Responses to partial nasal obstruction in sleeping infants. Aust Paediatr $\mathfrak{f}$ 1986; 22: 111-6.
3 Kemp JS, Thach BT. Sudden death in infants sleeping on polystyrene-filled cushions. $N$ Engl $\mathcal{f}$ Med 1991; 324 $1858-64$.

4 Cohle SD, Byard RW. Non-accidental death. In: Byard $\mathrm{RW}$, Cohle SD. Sudden death in infancy, childhood and adolescence. Cambridge: Cambridge University Press, 1994: 47-94.

5 Byard RW, Beal SM, Bourne AJ. Potentially dangerous infant sleeping environments. Arch Dis Child 1994; 71: 497-500.

6 Valdes-Dapena M, McFeeley PA, Hoffman HJ, et al. Histopathology atlas for the sudden infant death syndrome. Washington: Armed Forces Institute of Pathology, 1993.

7 McKenna J, Thomas EB, Anders TF, Sadeh A, Schechtman VL, Glotzbach SF. Infant-parent co-sleeping in an evolutionary perspective: implications for understanding infant sleep development and the sudden infant death syndrome. Sleep 1993; 16: 263-82.

8 Scragg R, Mitchell EA, Taylor BJ, et al. Bedsharing, smoking, and alcohol in the sudden infant death syndrome. $B M F$ 1993; 307: 1312-8.

9 Abramson $\mathrm{H}$. Accidental mechanical suffocation of infants. f Pediatr 1944; 25: 404-13.

10 Asphyxiation of neonate during breast feeding. Aust $\mathcal{f}$ Med 